\title{
Colaboradores de OUTRAS INSTituiÇÕes DE ENSINO
}

ABÍLIO DA SILVA GUERRA NETO

Universidade Presbiteriana Mackenzie - UPM

ALBERTO CIPINIUK

Pontifícia Universidade Católica do Rio de Janeiro - PUCRJ

ALDOMAR PEDRINI

Universidade Federal do Rio Grande do Norte - UFRN

ALESANDRO CASTROVIEJO

Universidade Presbiteriana Mackenzie - UPM

ANDREY ROSENTHAL SCHLEE

Universidade de Brasília - UnB

CAROLINA BIERRENBACH

Universidade Federal da Bahia - UFBA

ANA GABRIELA GODINHO LIMA

Universidade Presbiteriana Mackenzie - UPM

ANA LUCIA REIS MELO FERNANDES DA COSTA

Universidade Federal de Pernambuco - UFPE

ANA PAULA KOURY

Universidade São Judas Tadeu - USJT

ANA VAZ MILHEIRO

Instituto Universitário de Lisboa - ISCTE/Portugal

ANAT FALBEL

Universidade Estadual de Campinas - UNICAMP

ANDRÉA BORDE

Universidade Federal do Rio de Janeiro - UFRJ

ANGÉLICA TANUS B. ALVIM

Universidade Presbiteriana Mackenzie - UPM

ANTONIO CARLOS ZANI

Universidade Estadual de Londrina - UEL

ARLETE MOYSÉS RODRIGUES

Universidade Estadual de Campinas - UNICAMP

CARLOS ALBERTO FERREIRA MARTINS

Instituto de Arquitetura e Urbanismo - IAU/USP

CARLOS ANTÔNIO LEITE BRANDÃO

Universidade Federal de Minas Gerais - UFMG

CARLOS EDUARDO COMAS

Universidade Federal do Rio Grande do Sul - UFRGS

CARLOS GUILHERME MOTA

Universidade Presbiteriana Mackenzie - UPM

CARLOS ROBERTO M. DE ANDRADE

Instituto de Arquitetura e Urbanismo - IAU/USP

CAROLINA BORTOLOTTI

Universidade Estadual de Campinas - UNICAMP

CECÍLIA RODRIGUES DOS SANTOS

Universidade Presbiteriana Mackenzie - UPM

CÉLIA REGINA MORETTI MEIRELLES

Universidade Presbiteriana Mackenzie - UPM

CELSO LOMONTE MINOZZI

Universidade Presbiteriana Mackenzie - UPM

CLARA CORREIA D'ALAMBERT

Universidade de São Paulo - USP

CLÁUDIA CARVALHO

Fundação Casa de Rui Barbosa - FSCRB

CLÁUDIA NAVES DAVI AMORIM

Universidade de Brasília - UnB

CRISTINA MENEGUELLO

Universidade Estadual de Campinas - UNICAMP

DALILA ANDRADE DE OLIVEIRA

Universidade Federal de Minas Gerais - UFMG

DANIELE NUNES CAETANO DE SÁ

Pontifícia Universidade Católica DE Minas Gerais - PUCMG

DEBORAH MAGALHÃES LIMA

Universidade Federal de Minas Gerais - UFMG

DEMÉTRIO GUADAGNIN

Universidade Federal do Rio Grande do Norte UFRN

DENISE BARCELLOS PINHEIRO MACHADO

Universidade Federal do Rio de Janeiro - UFRJ

DORIS KOWALTOWSKY

Universidade Estadual de Campinas - UNICAMP

EDGARD DE ASSIS CARVALHO

Pontifícia Universidade Católica de São Paulo - PUCSP

EDSON DA CUNHA MAHFUZ

Universidade Federal do Rio Grande do Sul - UFRGS

ELOÍSA MAZZINI MIRANDA AUDI

Caixa Econômica Federal - CEF

EMMANUEL ANTONIO DOS SANTOS

Universidade do Vale do Paraíba - UNIVAP

ENEIDA MARIA SOUZA MENDONÇA

Universidade Federal do Espírito Santo - UFES

EUDES CAMPOS FILHO

Prefeitura Municipal de São Paulo - PMSP/DPH 
FÁBIO LOPES DE S. SANTOS

Instituto de Arquitetura e Urbanismo - IAU/USP

FERNANDO LARA

University of Texas at Austin, EUA

FERNANDO ATIQUE

Universidade Federal de São Paulo - UNIFESP

FERNANDO RUTTKAY PEREIRA

Universidade Federal de Santa Catarina - UFSC

FRANCISCO A. ROCCO LAHR

Instituto de Arquitetura e Urbanismo - IAU/USP

GENTIL PORTO FILHO

Universidade Federal da Bahia - UFPE

FRAYA FREHSE

Universidade de São Paulo - USP

GILDA COLLET BRUNA

Universidade Presbiteriana Mackenzie UPM

GIVALDO MEDEIROS

Instituto de Arquitetura e Urbanismo - IAU/USP

GLEICE AZAMBUJA ELALI

Universidade Federal do Rio Grande do Norte - UFRN

GUILHERME MAZZA DOURADO

Universidade de São Paulo - USP

HELENA NAPOLEON DEGREAS

Associação Paulista de Apoio à Familia - APAF

IRÃ JOSÉ TABORDA DUDEQUE

Universidade Tecnológica Federal do Paraná - UTFPR

IVONE SALGADO

Pontifícia Universidade Católica de Campinas - PUCCAMP

JOÃO LUIZ MUSA

Universidade de São Paulo - USP

JONATHAS MAGALHAES PEREIRA DA SILVA

Pontifícia Universidade Católica de Campinas - PUCCAMP

JOSÉ GERALDO SIMÕES JR

Universidade Presbiteriana Mackenzie - UPM

JOSE MANOEL MORALES SANCHEZ

Universidade de Brasília - UnB

JOUBERT JOSE LANCHA

Instituto de Arquitetura e Urbanismo - IAU/USP

JULIANA TORRES DE MIRANDA

Universidade Federal de Minas Gerais - UFMG
LAURA BUENO

Pontifícia Universidade Católica de Campinas - PUCCAMP

LEANDRO SILVA MEDRANO

Universidade Estadual de Campinas - UNICAMP

LÊDA M. BRANDÃO DE OLIVEIRA

Pesquisador Independente

LEILA MACEDO ODA

Fundação Oswaldo Cruz - FOC

LEONARDO BITTENCOURT

Universidade Federal do Rio Grande do Sul - UFRGS

LIA MAYUMI

Prefeitura Municipal de São Paulo - PMSPDHP

LINA FARIA

Universidade Federal do Rio de Janeiro - UFRJ

LIZETE MARIA RUBANO

Universidade Presbiteriana Mackenzie - UPM

LUCIANA COUTINHO

Universidade de Sorocaba - UNISO

LUDMILA BRANDÃO

Universidade Federal do Mato Grosso - UFMT

LUIS ESPALLARGAS GIMENEZ

Instituto de Arquitetura e Urbanismo - IAU/USP

LUIZ AMORIM

Universidade Federal de Pernambuco - UFPE

LUIZ CARLOS SOARES

Universidade Federal Fluminense - UFF

LUIZ GUILHERME RIVERA DE CASTRO

Universidade Presbiteriana Mackenzie - UPM

LUIZ MARQUES

Universidade Estadual de Campinas - UNICAMP

MANOELA RUFINONI

Universidade Federal de São Paulo - UNIFESP

MARCELO BEZERRA DE MELO TINOCO

Universidade federal do Rio grande do Norte - UFRN

MARCELO CLAUDIO TRAMONTANO

Instituto de Arquitetura e Urbanismo - IAU/USP

MÁRCIO COTRIM

Universidade Federal do Rio grande do Norte - UFRN

MÁRCIO MINTO FABRÍCIO

Instituto de Arquitetura e Urbanismo - IAU/USP 
MARCOS CARRILHO

Universidade Presbiteriana Mackenzie - UPM

MARGARETH AP.C.S. PEREIRA

Universidade Federal do Rio de Janeiro - UFRJ

MARIA ALICE JUNQUEIRA BASTOS

Pesquisador Independente

MARIA ANGELA DIAS

Universidade Federal do Rio de Janeiro - UFRJ

MARIA ANGELA P. CASTRO E S BORTOLUCCI

Instituto de Arquitetura e Urbanismo - IAU/USP

MARIA BEATRIZ CAMARGO CAPELLO

Universidade Federal de Uberlândia - UFU

MARIA BEATRIZ FURTADO RAHDE

Pontifícia Universidade Católica Rio Grande Sul - PUCRGS

MARIA CRISTINA WOLFF DE CARVALHO

Fundação Armando Álvares - FAAP

MARIA DA GLORIA LANCI SILVA

Universidade Salvador - UNIFACS

MARIA ELENA BERNARDES

Universidade Estadual de Campinas - UNICAMP

MARIA GABRIELA CELANI

Universidade Estadual de Campinas - UNICAMP

MARIA GABRIELA MARINHO

Universidade São Francisco - USF

MARIA INÊS SUGAI

Universidade Federal de Santa Catarina - UFSC

MARIA ISABEL VILLAC

Universidade Presbiteriana Mackenzie - UPM

MARIA LIGIA COELHO PRADO

Universidade de São Paulo - USP

MARIA PAULA ZAMBRANO FONTES

Fundação Oswaldo Cruz - FOC

MARIA LUIZA DE FREITAS

Universidade Federal de Pernambuco - UFPE

MARIA STELLA M. BRESCIANI

Universidade Estadual de Campinas - UNICAMP

MARIDALVA SOUZA PENTEADO

Universidade Estadual de Santa Cruz - UESC

MÁRIO MENDONÇA DE OLIVEIRA

Universidade Federal da Bahia - UFBA

MARIO THADEU LEME DE BARROS

Universidade de São Paulo - USP

MARLENE MILAN ACAYABA

Escritório Marcos Acayaba Arquitetos - SP

MAURO BARROS FILHO

Faculdade de Ciências Humanas - ESUDA
MIGUEL BUZZAR

Instituto de Arquitetura e Urbanismo - IAU/USP

MILTON GRANADO

Universidade Presbiteriana Mackenzie - UPM

MIRELLA DE MENEZES MIGLIARI

Escola Superior de Propaganda e Marketing - ESPM/RJ

MOZART ALBERTO BONAZZI DA COSTA

Pontifícia Universidade Católica de São Paulo - PUCSP

NADIA SOMEKH

Universidade Presbiteriana Mackenzie UPM

NADJA HERMANN

Pontifícia Universidade Católica Rio Grande Sul - PUCRGS

NEANDER FURTADO

Universidade de Brasília - UnB

NELSON BALTRUSIS

Universidade Católica do Salvador - UCSAL

OLGÁRIA MATTOS

Universidade de São Paulo - USP

OTÍLIA B. FIORI ARANTES

Universidade de São Paulo - USP

PATRICIA MAAS

Universidade Estadual Paulista - UNESP

PAULA DA CRUZ LANDIM

Universidade Estadual Paulista - UNESP

PAULO CHIESA

Universidade Federal do Paraná -UFPR

PAULO GARCEZ

Universidade de São Paulo - USP

PAULO KÜHL

Universidade Estadual de Campinas - UNICAMP

PAULO MARCOS BARNABÉ

Universidade Estadual de Londrina - UEL

PAULO YASSUHIDE FUJIOKA

Instituto de Arquitetura e Urbanismo - IAU/USP

PEDRO ARANTES

Universidade Federal de São Paulo - UNIFESP

PERCIVAL TIRAPELI

Universidade Estadual Paulista - UNESP

REGINA MARIA GONÇALVES BARCELLOS

Agência Nacional de Vigilância Sanitária - ANVISA

REGINA TIRELLO

Universidade Estadual de Campinas - UNICAMP

RENATA M. DE ALMEIDA

Universidade de São Paulo - USP

RENATO ANELLI

Instituto de Arquitetura e Urbanismo - IAU/USP 
RICARDO C CABÚS

Universidade Federal de Alagoas - UFAL

RICARDO TENA NUÑEZ

Escuela Superior de Ingenieria y Arquitectura - ESIA/México

ROBERTO BRAGA

Universidade Estadual Paulista - UNESP

ROBERTO LAMBERTS

Universidade Federal de Santa Catarina - UFSC

RODRIGO ALMEIDA BASTOS

Universidade Federal de Minas Gerais - UFMG

ROSINA TREVISAN

Universidade Federal do Rio de Janeiro - UFRJ

RUTH VERDE ZEIN

Universidade Presbiteriana Mackenzie - UPM

RUTHY NADIA LANIADO

Universidade Federal da Bahia - UFBA

SAIDE KAHTOUNI

Universidade São Judas Tadeu - USJT

SARAH FELDMAN

Instituto de Arquitetura e urbanismo - IAU/USP

SÉRGIO CONDE DE ALBITE SILVA

Universidade Federal do Estado do Rio de Janeiro - UFRJ

SÉRGIO FERRAZ MAGALHÃES

Universidade Federal do Rio de Janeiro - UFRJ

SERGIO LEUSIN

Universidade Federal Fluminense - UFF
SILVANA BARBOSA RUBINO

Universidade Estadual de Campinas - UNICAMP

SILVANA BERNARDES ROSA

Universidade do Estado de Santa Catarina - UDESC

SÍLVIA FICHER

Universidade de Brasília - UnB

SOLANGE FERRAZ DE LIMA

Universidade de São Paulo - USP

SONIA MARQUES

UniversidadeFederal do Rio grande do Norte - UFRN

SYLVIA CAIUBY NOVAES

Universidade de São Paulo - USP

SYLVIA DOBRY

Universidade Nove de Julho - UNINOVE

TELMA DE BARROS CORREIA

Instituto de Arquitetura e Urbanismo - IAU/USP

TERESA G. FLORENZANO

Instituto Nacional de Pesquisas Espaciais - INPE

pós-

VERA REGINA TÂNGARI

Universidade Federal do Rio de Janeiro - UFRJ

WILSON FLORIO

Universidade Presbieriana Mackenzie - UPM

WILSON RIBEIRO DOS SANTOS JR

Pontifícia Universidade Católica de Campinas - PUCCAMP

ZEULER LIMA

Sam Fox School - Washington University in St. Louis 